\title{
Bibliometric Analysis of Research Assessing the Use of Acupuncture for Pain Treatment Over the Past 20 Years
}

This article was published in the following Dove Press journal:

Journal of Pain Research

\author{
In-Seon Lee' \\ Hyangsook Lee (iD) ${ }^{1,2}$ \\ Yi-Hung Chen ${ }^{3}$ \\ Younbyoung Chae' \\ 'Acupuncture \& Meridian Science \\ Research Center, College of Korean \\ Medicine, Kyung Hee University, Seoul, \\ Republic of Korea; ${ }^{2}$ Korean Medicine \\ Convergence Research Center, Kyung \\ Hee University, Seoul, Republic of Korea; \\ ${ }^{3}$ Chinese Medicine Research Center, \\ China Medical University, Taichung, \\ Taiwan, R.O.C.
}

Objective: A bibliometric approach using quantitative analytical methods was applied to explore the development of research investigating acupuncture for the treatment of pain. This study also evaluated the current status of acupuncture analgesia with a visualization analysis of journal articles.

Methods: Articles on acupuncture for pain control that were published from 2000 to 2019 were retrieved from the Web of Science database. Extracted records were analyzed in terms of year of publication, country, journal, research area, authors, and organizational affiliations. The VOSviewer program was used to visualize trends in research on acupuncture for pain control.

Results: Analyses of 4595 original and review articles revealed that the total number of publications has continually increased over the last 20 years. The country producing the most articles in this field was the United States, followed by China and South Korea. A network analysis based on the cooccurrence of keywords revealed the following three major types of studies: clinical studies, pain management studies, and mechanism studies.

Conclusion: The present study evaluated research on acupuncture for pain control using bibliometric methods and revealed current trends in acupuncture analgesia research, as well as potential future hot spots of research in this field.

Keywords: acupuncture, analgesia, bibliometric analysis, pain, network analysis

\section{Introduction}

Acupuncture is one of the most commonly used complementary and alternative medicines, and has been widely applied for the treatment of various conditions, including pain, stroke, nausea and vomiting, and for management of mental health. ${ }^{1,2}$ Acupuncture research has markedly increased during the past two decades and it is now widely used for pain management. ${ }^{1}$ Because acupuncture is efficacious for pain management, ${ }^{3}$ many of the mechanisms underlying acupuncture analgesia have been explored. ${ }^{4}$ Several clinical trials have demonstrated the efficacy of acupuncture for chronic back pain ${ }^{5}$ and headache. ${ }^{6}$ Furthermore, many studies have assessed the underlying mechanisms of acupuncture analgesia in humans using brain imaging modalities, ${ }^{7-9}$ and in animals using neurochemical approaches. ${ }^{10,11}$ However, despite the rapid increase in the number of published studies and important findings regarding the effects and mechanisms of acupuncture analgesia, there has been little quantitative analysis of research on acupuncture for pain control in terms of the impact of articles (based on the citation index), authors and organizational affiliations.
Correspondence: Younbyoung Chae Acupuncture \& Meridian Science Research Center, Kyung Hee University, I Hoegi-dong, Dongdaemun-gu, Seoul, Republic of Korea

Email ybchae@khu.ac.kr 
Bibliometrics is a quantitative analysis method that uses mathematical and statistical tools to measure the interrelationships and impacts of publications within a given area of research. This method can provide a macroscopic overview of large amounts of academic literature, and can also be used to efficiently identify influential studies, authors, journals, organizations, and countries over time. ${ }^{12}$ Recently, a network analysis that employed bibliometric tools identified established and emerging research areas more intuitively by mapping social networks using co-word, co-authorship, and co-citation analyses. ${ }^{13,14}$ Bibliometric analyses have also been utilized to assess research trends in a variety of fields, such as pain and nursing, ${ }^{15}$ breast cancer, ${ }^{16}$ diabetes mellitus and T cells, ${ }^{17}$ and spinal ultrasound; ${ }^{18}$ such analyses can provide data to inform policy-making and clinical guidelines. Several bibliometric studies of general trends in acupuncture research have been conducted. ${ }^{1,4,19-21}$ For example, Ma et al evaluated all acupuncture-related research over the past 20 years and summarized the types of studies, health conditions assessed, countries of origin, journals and impact factors. ${ }^{1}$ Li et al performed a similar assessment of acupuncture research in Taiwan, ${ }^{20}$ and Liang et al performed bibliometric analyses of studies of acupuncture treatment for low back pain. ${ }^{21}$ However, there has been little study of overall trends in acupuncture analgesia research based on bibliometric analyses.

Thus, the present study used a bibliometric approach to analyze patterns in research on acupuncture for pain control, and to evaluate the current state of research on acupuncture analgesia.

\section{Methods}

\section{Sources of Data and Search Strategy}

All data were retrieved from the Web of Science via the China Medical University Library website on July 28, 2019 using the following search terms: (acupuncture OR electroacupuncture) AND (pain). The Web of Science provides comprehensive publication data and is the widely accepted and frequently used database for the analysis of scientific publications. The publication period considered in this study was from January 1, 2000 to July 28, 2019; a total of 5230 articles were identified. Articles that were not original articles or reviews ( $\mathrm{n}=484)$, and those that were not written in English were excluded $(n=151)$; thus, a total of 4595 articles were ultimately included in the final analyses.

\section{Data Analysis}

Publications were stratified and systematically assessed according to publication year, country, journal, research area, authors, and organizational affiliations. Additionally, the frequencies of keywords extracted from the articles were assessed and then included in a network analysis of the development of research on acupuncture for pain control.

All data were downloaded from the Web of Science and imported into VOSviewer v.1.6.11 (Centre for Science and Technology Studies, Leiden University, Leiden, The Netherlands), which is commonly used to analyze and visualize relationships among authors, countries, co-citations, and the terms used in articles. ${ }^{12,22}$ The visualization of similarities

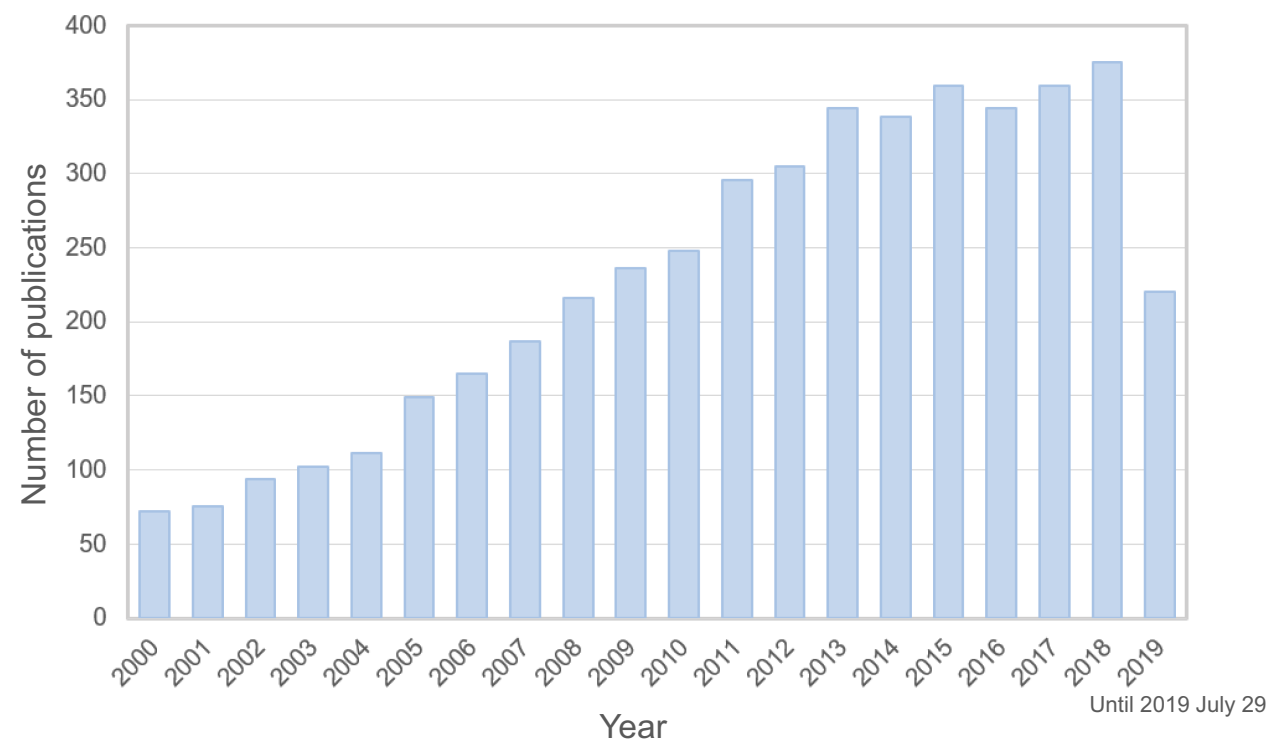

Figure I Number of publications investigating acupuncture and pain by year, over the past 20 years. 
Table I The 15 Countries Producing the Highest Number of Original and Review Articles Investigating Acupuncture for Pain Control

\begin{tabular}{|l|l|l|l|}
\hline & Country & Records (n) & \% of (4595) \\
\hline 1 & USA & 1412 & 30.7 \\
2 & Peoples R China & 1111 & 24.2 \\
3 & South Korea & 476 & 10.4 \\
4 & UK & 443 & 9.6 \\
5 & Germany & 310 & 6.7 \\
6 & Australia & 239 & 5.2 \\
7 & Taiwan & 214 & 4.7 \\
8 & Canada & 201 & 4.4 \\
9 & Brazil & 171 & 3.7 \\
10 & Sweden & 139 & 3.0 \\
11 & Japan & 128 & 2.8 \\
12 & Italy & 109 & 2.4 \\
13 & Turkey & 71 & 1.5 \\
14 & Switzerland & 70 & 1.5 \\
15 & Austria & 65 & 1.4 \\
\hline
\end{tabular}

Table 2 The 15 Journals Publishing the Highest Number of Original and Review Articles Investigating Acupuncture for Pain Control

\begin{tabular}{|c|l|l|l|}
\hline & Journal Title & $\begin{array}{l}\text { Records } \\
\text { (n) }\end{array}$ & \% (of 4595) \\
\hline 1 & $\begin{array}{l}\text { Evidence Based Complementary and } \\
\text { Alternative Medicine }\end{array}$ & 357 & 7.8 \\
\hline 2 & $\begin{array}{l}\text { Journal of Alternative and Complementary } \\
\text { Medicine }\end{array}$ & 239 & 5.2 \\
\hline 3 & Acupuncture in Medicine & 224 & 4.9 \\
\hline 4 & Complementary Therapies in Medicine & 120 & 2.6 \\
\hline 5 & $\begin{array}{l}\text { BMC Complementary and Alternative } \\
\text { Medicine }\end{array}$ & 96 & 2.1 \\
\hline 6 & Trials & 88 & 1.9 \\
\hline 7 & Cochrane Database of Systematic Reviews & 83 & 1.8 \\
\hline 8 & American Journal of Chinese Medicine & 76 & 1.7 \\
\hline 9 & Medicine & 69 & 1.5 \\
\hline 10 & PLoS One & 69 & 1.5 \\
\hline 11 & Acupuncture Electro Therapeutics Research & 66 & 1.3 \\
\hline 12 & Pain & 62 & 1.3 \\
\hline 13 & Clinical Journal of Pain & 61 & \\
\hline 14 & European Journal of Integrative Medicine & 54 & 1.2 \\
\hline 15 & Pain Medicine & 63 & \\
\hline
\end{tabular}

(VOS) mapping method was used to estimate similarity (affinity) according to association strength, where higher association strength is indicative of greater similarity between terms, and a larger number of publications in which two items cooccur indicates that the terms are more closely similar to each other. The number of clusters can be varied depending on threshold of similarity between the nodes. The resolution of clustering was set as the default value (1.00) in this study. ${ }^{22}$

Additionally, keywords were analyzed to identify popular topics in research on acupuncture for pain control. Keywords indicate article research themes; cooccurring keywords reveal associations in underlying themes among articles. Co-words were defined as words cooccurring in the titles or abstracts of the articles identified herein. Keywords were defined as words used more than 80 times in titles and abstracts across all publications. The VOS method was applied to cluster keywords into different groups; each cluster was identified with a different color. Each word is represented by a circle, the diameter and label size of which denote the

Table 3 The 15 Most Well-Represented Research Areas in Terms of the Number of Original and Review Articles Investigating Acupuncture for Pain Control

\begin{tabular}{|c|l|l|l|}
\hline & Research Area & $\begin{array}{l}\text { Records } \\
\text { (n) }\end{array}$ & \% (of 4595) \\
\hline 1 & $\begin{array}{l}\text { Integrative Complementary } \\
\text { Medicine }\end{array}$ & 1530 & 33.3 \\
\hline 2 & Neurosciences Neurology & 899 & 19.6 \\
\hline 3 & General Internal Medicine & 586 & 12.8 \\
\hline 4 & Anesthesiology & 336 & 7.3 \\
\hline 5 & Rehabilitation & 250 & 5.4 \\
\hline 6 & Research Experimental Medicine & 224 & 4.9 \\
\hline 7 & Orthopedics & 163 & 3.5 \\
\hline 8 & Health Care Sciences Services & 146 & 3.2 \\
\hline 9 & Obstetrics Gynecology & 132 & 2.9 \\
\hline 10 & Rheumatology & 127 & 2.8 \\
\hline 11 & Oncology & 124 & 2.7 \\
\hline 12 & Sport Sciences & 118 & 2.6 \\
\hline 13 & Pharmacology Pharmacy & 110 & 2.4 \\
\hline 14 & Science Technology Other Topics & 106 & 2.3 \\
\hline 15 & Nursing & 105 & 2.3 \\
\hline
\end{tabular}




\section{A}

\section{Cluster 1 (clinical studies)}

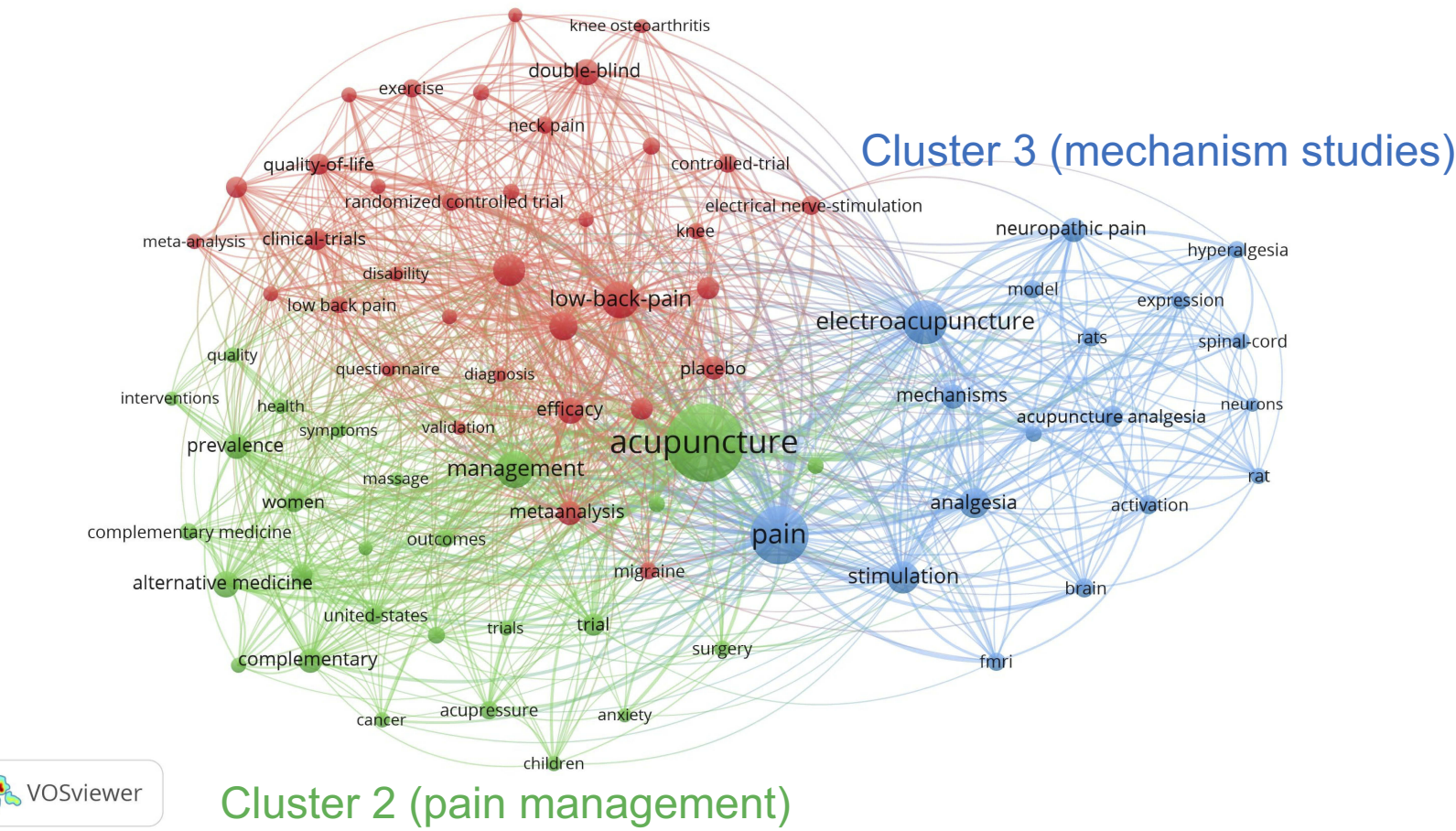

\section{B}

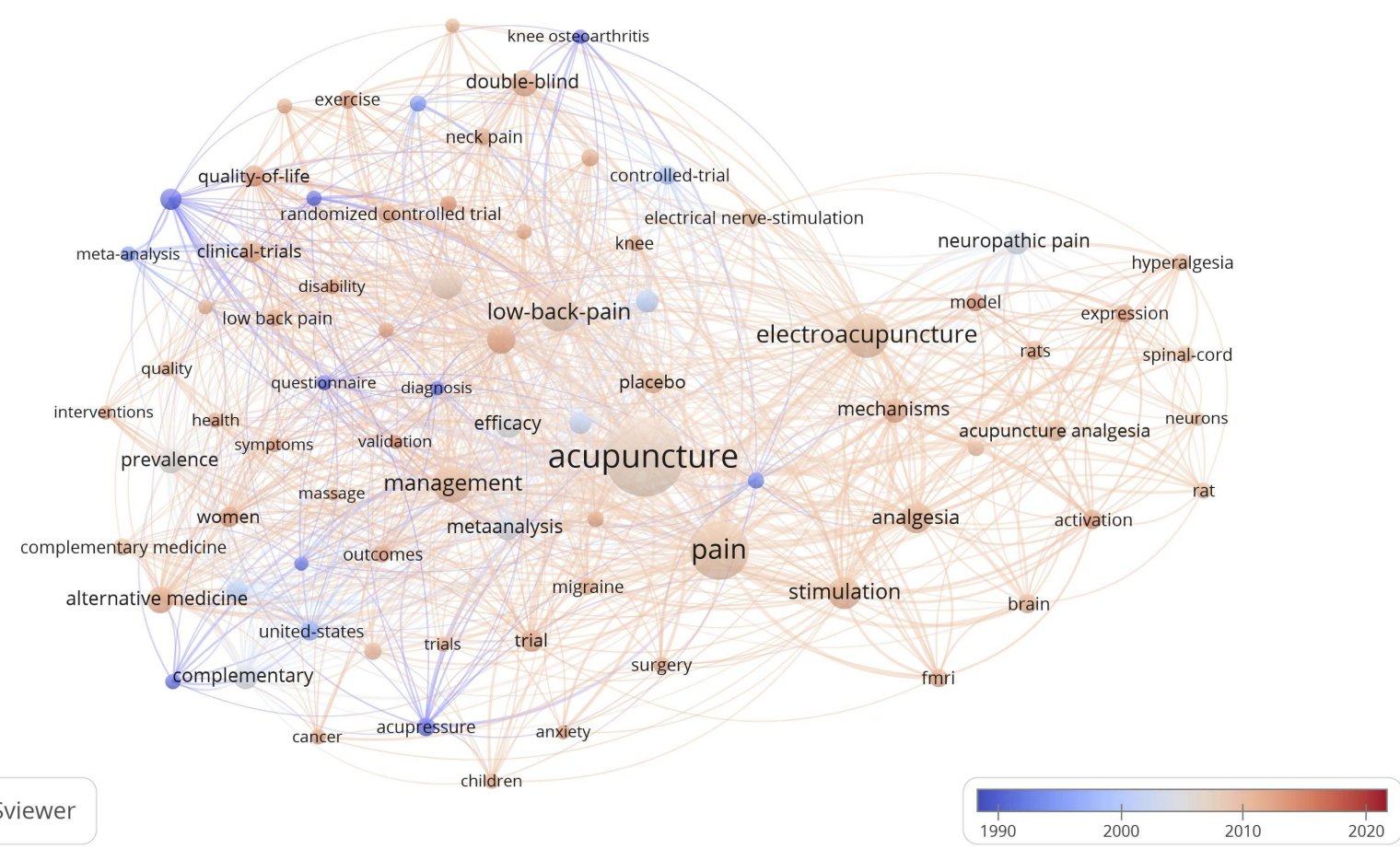

Figure 2 Analysis of keywords. (A) Mapping of keywords of studies pertaining to acupuncture and pain (categorized into three groups). (B) Distribution of keywords according to average publication year (blue: earlier, red: later).

number of occurrences in titles or abstracts. Colors represent groups of linked terms; the label size of a term represents the number of publications on acupuncture for pain control in which it is used, and the distance between two terms represents the degree to which they are associated. 


\section{Results}

\section{Global Trends in Studies Investigating Acupuncture for Pain Control Over the Past 20 Years}

The number of articles related to acupuncture for pain control has steadily increased over the past 20 years, but with some fluctuations among years (Figure 1). Of the 54 countries identified in the present study, the United States had the most publications $(30.7 \%)$, followed by China (24.2\%) and South Korea (10.4\%; Table 1).

\section{Analysis of Journals and Research Areas}

Of the 900 journals that were identified in the present study, Evidence-based Complementary and Alternative Medicine published the most articles (7.8\%), followed by the Journal of Alternative and Complementary Medicine (5.2\%) and Acupuncture in Medicine (4.9\%; Table 2).

Of the 71 research areas that were identified in the present study, the most well-represented in terms of number of articles was integrative complementary medicine (33.3\% of all articles), followed by neuroscience, neurology $(19.6 \%)$, general internal medicine $(12.8 \%)$, and anesthesiology (7.3\%; Table 3$)$.

\section{Analysis of Keywords}

The keywords in the 4595 publications assessed in the present study were analyzed using VOSviewer (Figure 2). A total of 80 keywords were identified as having occurred more than 80 times in the title and abstract fields across all articles. These keywords appeared a total of 10,941 times across all publications, where the studies were classified as clinical studies, pain management studies, and mechanism studies. In the clinical studies cluster (Cluster 1), 39 keywords were frequently used, including acupuncture (2394 times), low back pain (550 times), management (545 times), randomized controlled trial (408 times), therapy (310 times), double-blind (280 times), and efficacy (280 times). In the pain management cluster (Cluster 2), 23 keywords were frequently used, including alternative medicine (283 times), prevalence (255 times), complementary (239 times), trial (192 times), care (181 times), and women (159 times). In the mechanism studies cluster (Cluster 3), 18 keywords were frequently used, including pain (1337 times), electro-acupuncture (759 times), stimulation (408 times), analgesia (367 times), mechanisms (237 times), neuropathic pain (233 times), and acupuncture analgesia (173 times).
The keywords were color-coded by VOSviewer based on average publication year, where blue indicates that a keyword appeared earlier and red indicates that a keyword appeared later.

\section{Analysis of Authors}

Table 4 shows the most top 15 frequent authors, according to the author lists of the articles included in the present study. Hyejung Lee of Kyung Hee University, South Korea authored or co-authored the most articles (69 articles) followed by Lixing Lao of Hong Kong University (65 articles) and Myeongsoo Lee of the Korea Institute of Oriental Medicine (KIOM; 65 articles).

Table 4 Top 15 Authors with Respect to the Number of Original and Review Articles Investigating Acupuncture for Pain Control

\begin{tabular}{|c|l|l|l|}
\hline & Author & $\begin{array}{l}\text { Records } \\
\text { (n) }\end{array}$ & $\%$ (of 4595) \\
\hline 1 & $\begin{array}{l}\text { Lee H (Kyung Hee University, South } \\
\text { Korea) }\end{array}$ & 69 & 1.5 \\
\hline 2 & Lao LX (Maryland University, USA) & 65 & 1.4 \\
\hline 3 & $\begin{array}{l}\text { Lee MS (Korea Institute of Oriental } \\
\text { Medicine) }\end{array}$ & 65 & 1.4 \\
\hline 4 & $\begin{array}{l}\text { Lee JH (Seoul National University, } \\
\text { South Korea) }\end{array}$ & 61 & 1.3 \\
\hline 5 & $\begin{array}{l}\text { Macpherson H (University of York, } \\
\text { United Kingdom) }\end{array}$ & 59 & 1.3 \\
\hline 6 & $\begin{array}{l}\text { Park HJ (Kyung Hee University, South } \\
\text { Korea) }\end{array}$ & 57 & 1.2 \\
\hline 7 & $\begin{array}{l}\text { Ernst E (University of Exeter, United } \\
\text { Kingdom) }\end{array}$ & 55 & 1.2 \\
\hline 8 & Kaptchuk TJ (Harvard University, USA) & 54 & 1.2 \\
\hline 9 & Yang J (Chengdu University, China) & 45 & 1.0 \\
\hline 10 & $\begin{array}{l}\text { Witt CM (University of Zurich, } \\
\text { Switzerland) }\end{array}$ & 42 & 0.9 \\
\hline 11 & $\begin{array}{l}\text { Chae Y (Chengdu University, South } \\
\text { Korea) }\end{array}$ & 41 & 0.9 \\
\hline 12 & Liang FR (Cheugdu University, China) & 40 & 0.9 \\
\hline 13 & Berman BM (Maryland University, USA) & 39 & 0.8 \\
\hline 14 & $\begin{array}{l}\text { Shin BC (Pusan National University, } \\
\text { South Korea) }\end{array}$ & 39 & 0.8 \\
\hline LinJG (China Medical University, Taiwan) & 37 & 0.8 \\
\hline
\end{tabular}


The authors of the 4595 publications were analyzed using VOSviewer (Figure 3). The results showed that 59 authors appeared more than 15 times (out of 16577 authors); these authors were classified into seven clusters: Cluster 1, 12 authors from the United Kingdom or Germany, including Claudia Witt (2138 citations), Klaus Linde (1971 citations), Hugh Macpherson (1706 citations), and Benno Brinkhaus (1540 citations); Cluster 2, 11 authors from China, including Jie Tian (636 citations), Wei Qin (610 citations), and Lijun Bai (515 citations); Cluster 3, 6 authors from the KIOM and collaborators, including Myeong soo Lee (1084 citations), Edzard Ernst (726 citations), and Sun-Mi Choi (375 citations); and Cluster 4, 4 authors from Harvard Medical School, including Ted
Kaptchuk (1475 citations), Vitaly Napadow (1342 citations), Jian Kong (1247 citations), and Randy Gollub (858 citations).

Authors were color-coded by VOSviewer based on the number of citations; blue indicates that the authors were cited a low number of times and red indicates that they were cited a high number of times.

\section{Analysis of Organizational Affiliations}

Table 5 shows the top 15 organizational affiliations of the 4595 articles identified in the present study. Kyung Hee University published the most articles (5.6\%), followed by Harvard University (4.2\%). The organizational affiliations of the publications were analyzed using VOSviewer (Figure 4), which

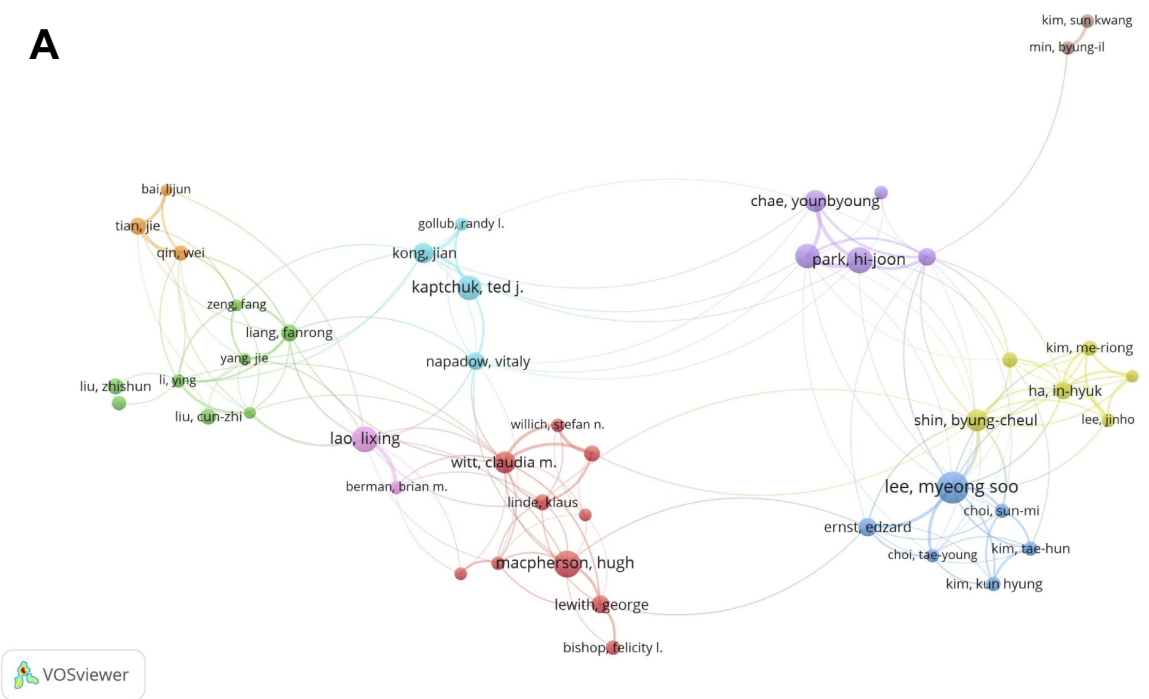

B

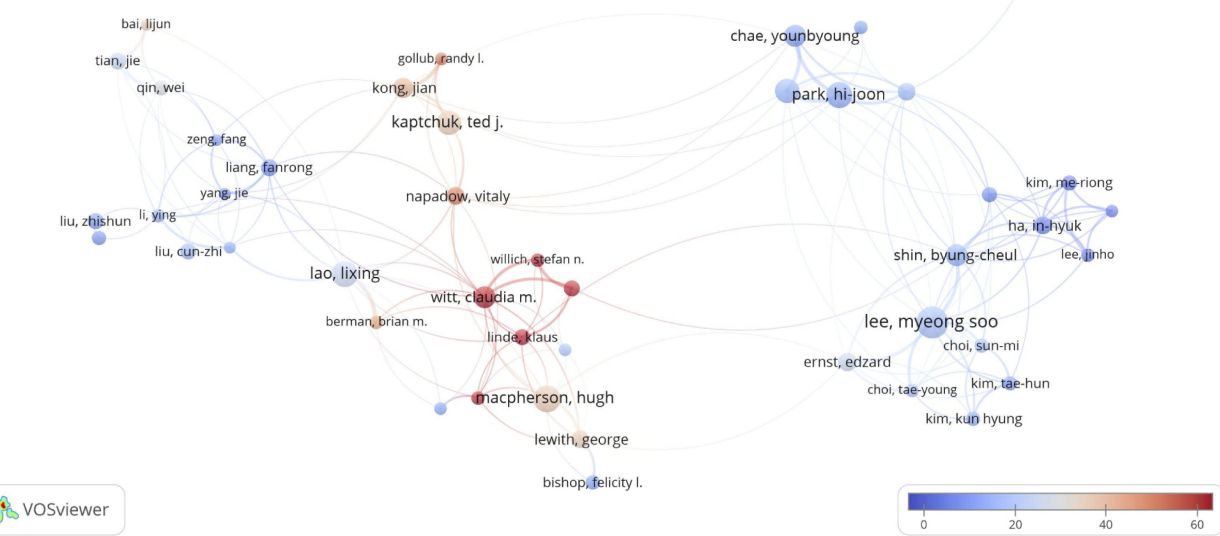

Figure 3 Network map of study authors. (A) Of the 16,577 authors (classified into seven clusters), 46 had at least I5 publications. (B) Distribution of authors according to citations. 
revealed that 53 organizations each published more than 30 articles (total of 3899). The organizations were classified into seven clusters: Cluster 1, 14 organizations including Harvard University (7147 citations), Massachusetts General Hospital (4728 citations), and the University of Washington (2311 citations); Cluster 2, 11 organizations including Peking University (1642 citations), the University of Florida (1534 citations), and Capital Medical University (1015 citations); Cluster 3; eight organizations including Kyung Hee University (4333 citations), the University of Exeter (3329 citations), and the University of Plymouth (3144 citations); and Cluster 4, four organizations including the Technical University of Munich (3053 citations), the University of York (2032 citations), Memorial Sloan Kettering Cancer Center (1845 citations), and the University of Southampton (1690 citations).

The organizations were color-coded by VOSviewer based on the number of publications with which they were affiliated; blue indicates a low number of affiliations and red indicates a high number of affiliations.

Table 5 Top 15 Organizations with Respect to the Number of Original and Review Articles Investigating Acupuncture for Pain Control

\begin{tabular}{|c|l|l|l|}
\hline & Organization & $\begin{array}{l}\text { Records } \\
\text { (n) }\end{array}$ & \% (of 4595) \\
\hline 1 & Kyung Hee University & 255 & 5.6 \\
\hline 2 & Harvard University & 195 & 4.2 \\
\hline 3 & VA Boston Healthcare System & 144 & 3.1 \\
\hline 4 & University of California System & 124 & 2.7 \\
\hline 5 & $\begin{array}{l}\text { Korea Institute of Oriental } \\
\text { Medicine KIOM }\end{array}$ & 123 & 2.7 \\
\hline 6 & $\begin{array}{l}\text { China Academy of Chinese } \\
\text { Medical Sciences }\end{array}$ & 120 & 2.6 \\
\hline 7 & $\begin{array}{l}\text { Beijing University of Chinese } \\
\text { Medicine }\end{array}$ & 108 & 2.4 \\
\hline 8 & China Medical University Taiwan & 108 & 2.4 \\
\hline 9 & University System of Maryland & 103 & 2.2 \\
\hline 10 & University of Maryland Baltimore & 100 & 2.2 \\
\hline 11 & Massachusetts General Hospital & 92 & 2.0 \\
\hline 12 & University of Texas System & 82 & 1.8 \\
\hline 13 & University of Exeter & 79 & 1.7 \\
\hline 14 & Capital Medical University & 77 & 1.7 \\
\hline 15 & Fudan University & 76 & 1.7 \\
\hline
\end{tabular}

\section{Discussion}

The present bibliometric analysis of studies investigating acupuncture for pain control published during the last two decades revealed that the total number of articles steadily increased during this period. The largest proportion of articles in this area were from the United States, followed by China and South Korea. The most well-represented fields of study in terms of number of articles were integrative complementary medicine, neuroscience, general internal medicine, and anesthesiology. Additionally, a network analysis based on the co-occurrence of keywords revealed three distinct types of studies, i.e., clinical studies, pain management studies, and mechanism studies. The present findings may be useful for organizing and prioritizing future research on acupuncture analgesia.

Keywords can provide information about the core content of an article, and can also be used to identify research trends in a particular domain. In the present study, analysis of keywords revealed three study types (clinical, pain management, and mechanism studies; Figure 2A), as discussed above. Many clinical trials have investigated the efficacy of acupuncture for patients with low back pain or neck pain (Cluster 1: clinical studies) and, as a well-known complementary and alternative medicine, acupuncture treatment is now widely used to treat cancer-related and postoperative pain as an alternative to opioids (Cluster 2: pain management). ${ }^{23,24}$ Furthermore, many studies, including brain imaging studies of humans and animals, have explored the neural substrates of acupuncture associated with pain control (Cluster 3: mechanism studies). ${ }^{9,10}$ The present findings indicate that these study types together account for the majority of research on the effects and mechanisms of acupuncture treatment for pain control. Furthermore, based on our analysis of the changes in keywords by publication year, mechanism study keywords (Cluster 3) have recently become more prevalent than the keywords of the other study types (Figure 2B).

Our analyses of authors and organizational affiliations revealed that many research groups in Asian countries have published a large number of studies on acupuncture for pain control. However, the authors with the most citations were from Europe and the United States, such as Claudia Witt, Klaus Linde, Benno Brinkhaus, and Hugh Macpherson (Europe) and Ted Kaptchuk, Vitaly Napadow, and Jian Kong (United States). However, citations might not be the best index of publication quality; although a considerable number of articles were from Asian countries, improvement in the quality of these publications is necessary. 
Although we acknowledge that there may be an overlap between this study and previous studies, ${ }^{1,4,19-21}$ the present research makes a novel contribution in terms of the network analysis of keywords. For example, Ma et al did not include network analysis of keywords, authors, and/or organizations, although they included all acupuncture-related research articles $(\mathrm{n}=13320) .{ }^{1}$ Literatures related with acupuncture for low back pain $(n=958)$ were analyzed with respect to country/institution, cited journals, authors/cited authors, cited references, and keywords and visualized with CiteSpace tool. ${ }^{21}$ In the current study, we included literatures with regards to acupuncture for pain management including both clinical and animal studies $(\mathrm{n}=4595)$ to provide global trends in acupuncture in pain control. Our study will contribute to supporting the current use of acupuncture in pain control as an alternative to opioids. Most of previous studies commonly found that top-ranked countries with respect to numbers of acupuncture-related publications are China, United States, South Korea, United Kingdom and Germany. ${ }^{1,4,21}$ In the early stage of publications, Western countries including United States and Germany led the acupuncture research and conducted many clinical trials while nowadays Eastern countries including China and South Korea conducted many clinical trials as well as mechanism studies.
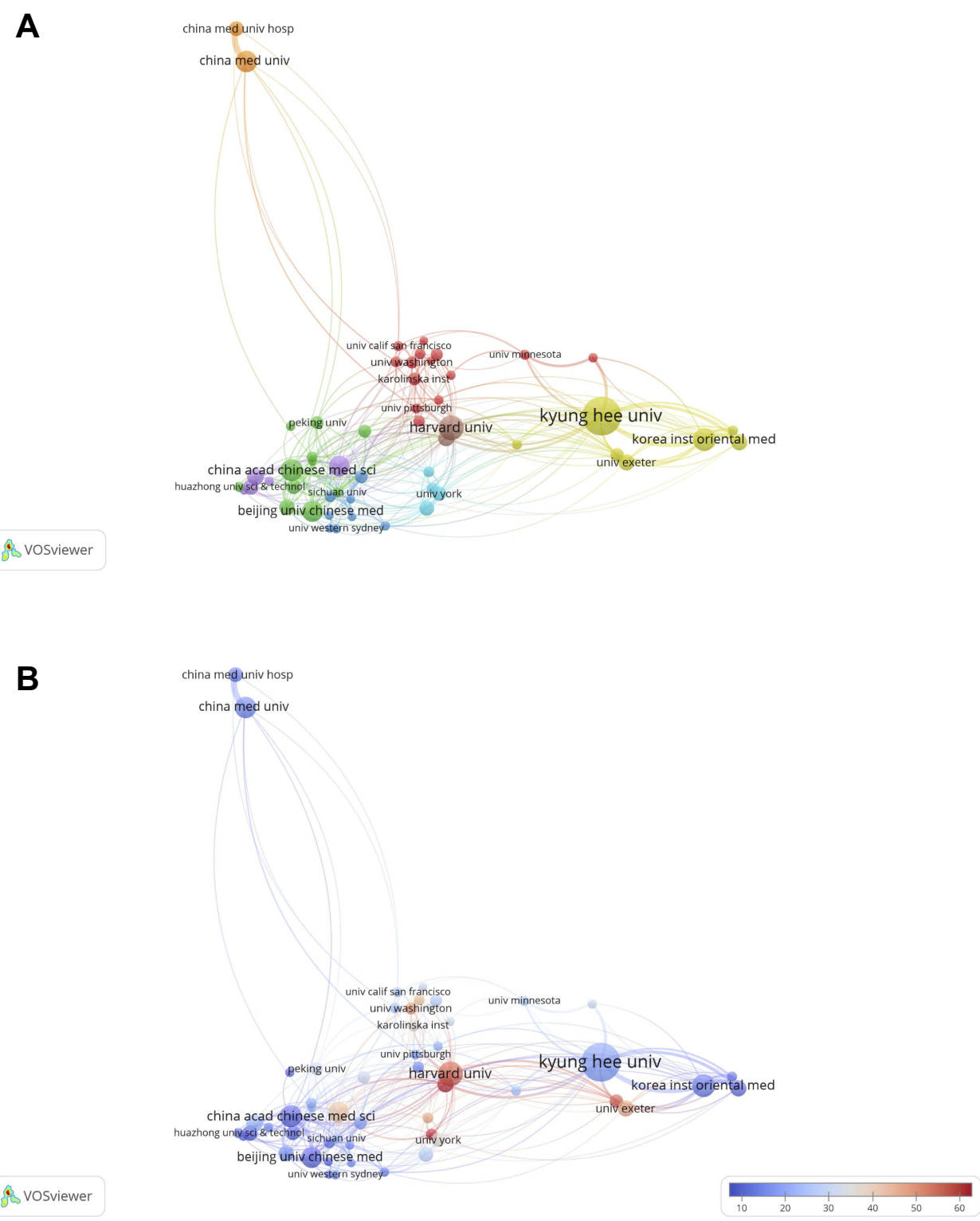

Figure 4 Network map of organizational affiliations. (A) Of the 3899 organizations (classified into seven clusters), 53 were affiliated with at least 30 publications. (B) Distribution of organizations according to citations. 
This study has some limitations. Web of Science covers other languages, but we only included English language in this study. Since other types of language were about $3 \%$ of the total articles, it is expected that the overall trends of our results might be similar to the results from without language restrictions. Furthermore, the number of clusters and the label of clusters in the network analysis can be varied based on the resolution of clustering and authors' subjective view. The last issue is that the VOSviewer uses the original affiliations which might not differ associated organizations precisely. For example, Massachusetts General Hospital, which is closely affiliated with Harvard Medical School, and Harvard University are separately analyzed in this study.

In conclusion, the present study used bibliometric methods to identify research trends in the field of acupuncture for pain control over the past two decades. Additionally, a network analysis revealed three main study types (i.e., clinical, pain management, and mechanism studies) with respect to acupuncture research for pain management. These results also provide insight into trends in acupuncture analgesia research and potential future hot spots of research in this field.

\section{Acknowledgments}

This research was supported by Basic Science Research Program through the National Research Foundation of Korea (NRF) funded by the Ministry of Science, ICT \& Future Planning (No. 2018R1D1A1B07042313 and No. 2018R1A6A6031080). We would like to thank Ms. Iona MacDonald for assisting with proofreading.

\section{Disclosure}

The authors declare that they have no conflicts of interest regarding this paper and that the funders had no role in study design, data collection and analysis, decision to publish, or preparation of the manuscript.

\section{References}

1. Ma Y, Dong M, Zhou K, Mita C, Liu J, Wayne PM. Publication trends in acupuncture research: a 20-year bibliometric analysis based on PubMed. PLoS One. 2016;11(12):e0168123. doi:10.1371/journal.pone.0168123

2. Wang H, Yang G, Wang S, Zheng X, Zhang W, Li Y. The most commonly treated acupuncture indications in the United States: a cross-sectional study. Am J Chin Med. 2018;46:1-33.

3. Vickers AJ, Vertosick EA, Lewith G, et al. Acupuncture for chronic pain: update of an individual patient data meta-analysis. $J$ Pain. 2018;19(5):455-474. doi:10.1016/j.jpain.2017.11.005

4. Han JS, Ho YS. Global trends and performances of acupuncture research. Neurosci Biobehav Rev. 2011;35(3):680-687. doi:10.1016/j. neubiorev.2010.08.006
5. Berman BM, Langevin HM, Witt CM, Dubner R. Acupuncture for chronic low back pain. $N$ Engl $J$ Med. 2010;363(5):454-461. doi:10.1056/NEJMct0806114

6. Linde K, Streng A, Jurgens S, et al. Acupuncture for patients with migraine: a randomized controlled trial. JAMA. 2005;293(17):21182125. doi:10.1001/jama.293.17.2118

7. Chae Y, Chang DS, Lee SH, et al. Inserting needles into the body: a meta-analysis of brain activity associated with acupuncture needle stimulation. J Pain. 2013;14(3):215-222. doi:10.1016/j.jpain.2012.11.011

8. Huang W, Pach D, Napadow V, et al. Characterizing acupuncture stimuli using brain imaging with FMRI-a systematic review and meta-analysis of the literature. PLoS One. 2012;7(4):e32960. doi:10.1371/journal.pone. 0032960

9. Maeda Y, Kim H, Kettner N, et al. Rewiring the primary somatosensory cortex in carpal tunnel syndrome with acupuncture. Brain. 2017;140(4):914-927. doi:10.1093/brain/awx015

10. Chen YH, Lee HJ, Lee MT, et al. Median nerve stimulation induces analgesia via orexin-initiated endocannabinoid disinhibition in the periaqueductal gray. Proc Natl Acad Sci U S A. 2018;115(45): E10720-E29. doi:10.1073/pnas.1807991115

11. Lin JG, Chen WL. Acupuncture analgesia: a review of its mechanisms of actions. Am J Chin Med. 2008;36(4):635-645. doi:10.1142/ S0192415X08006107

12. van Eck NJ, Waltman L. Software survey: VOSviewer, a computer program for bibliometric mapping. Scientometrics. 2010;84 (2):523-538. doi:10.1007/s11192-009-0146-3

13. Fonseca B, Silva M, Araujo KM, Sampaio RB, Moraes MO. Network analysis for science and technology management: evidence from tuberculosis research in Fiocruz, Brazil. PLoS One. 2017;12(8): e0181870. doi: 10.1371 journal.pone. 0181870

14. Koo M. A bibliometric analysis of two decades of aromatherapy research. BMC Res Notes. 2017;10(1):46. doi:10.1186/s13104-016-2371-1

15. Damar HT, Bilik O, Ozdagoglu G, Ozdagoglu A, Damar M. Scientometric overview of nursing research on pain management. Rev Lat Am Enfermagem. 2018;26:e3051. doi:10.1590/1518-8345.2581.3051

16. Wang Y, Zhai X, Liu C, Wang N, Wang Y. Trends of triple negative breast cancer research (2007-2015): a bibliometric study. Medicine (Baltimore). 2016;95(46):e5427. doi:10.1097/MD.0000000000005427

17. Gao Y, Wang Y, Zhai X, et al. Publication trends of research on diabetes mellitus and T cells (1997-2016): a 20-year bibliometric study. PLoS One. 2017;12(9):e0184869. doi:10.1371/journal.pone.0184869

18. Zhai X, Cui J, Shao J, et al. Global research trends in spinal ultrasound: a systematic bibliometric analysis. BMJ Open. 2017;7(10): e015317. doi:10.1136/bmjopen-2016-015317

19. Kung YY, Hwang SJ, Li TF, Ko SG, Huang CW, Chen FP. Trends in global acupuncture publications: an analysis of the Web of science database from 1988 to 2015. J Chin Med Assoc. 2017;80(8):521-525. doi:10.1016/j.jcma.2017.01.010

20. Li TF, Kung YY, Tsai CH, Hwang SJ, Chen FP. A bibliometric analysis of acupuncture research in Taiwan from 1988 to 2017. J Chin Med Assoc. 2019;82(5):428-435. doi:10.1097/JCMA.0000000000000093

21. Liang YD, Li Y, Zhao J, Wang XY, Zhu HZ, Chen XH. Study of acupuncture for low back pain in recent 20 years: a bibliometric analysis via CiteSpace. J Pain Res. 2017;10:951-964. doi:10.2147/JPR.S132808

22. van Eck NJ, Waltman L. Citation-based clustering of publications using CitNetExplorer and VOSviewer. Scientometrics. 2017;111 (2):1053-1070. doi:10.1007/s11192-017-2300-7

23. Heyward J, Jones CM, Compton WM, et al. Coverage of nonpharmacologic treatments for low back pain among US public and private insurers. JAMA Netw Open. 2018;1(6):e183044. doi:10.1001/jamanetworkopen. 2018.3044

24. Kong JT. Exploring the multiple roles of acupuncture in alleviating the opioid crisis. J Altern Complement Med. 2018;24(4):304-306. doi:10.1089/acm.2018.0083 


\section{Publish your work in this journal}

The Journal of Pain Research is an international, peer reviewed, open access, online journal that welcomes laboratory and clinical findings in the fields of pain research and the prevention and management of pain. Original research, reviews, symposium reports, hypothesis formation and commentaries are all considered for publication. The manuscript management system is completely online and includes a very quick and fair peer-review system, which is all easy to use. Visit http:// www.dovepress.com/testimonials.php to read real quotes from published authors. 